\title{
Prevalência de transtornos mentais não-psicóticos e fatores associados em pessoas com hipertensão arterial sistêmica e/ou diabetes mellitus em Unidades de Saúde da Família em Blumenau, Santa Catarina
}

Prevalence of non-psychotic mental disorders and associated factors in people with hypertension and/or diabetes from Family Health Units in Blumenau, Santa Catarina, Brazil Ernani Tiaraju de Santa Helena' ${ }^{1}$ Bruno Gaiarsa Simões Lasagno², Ramiro Vieira ${ }^{2}$

Palavras-chave: Transtornos Mentais Atenção Primária à Saúde Peneiramento Mental Disorders Primary Health Care

Straining

\section{Resumo}

Objetivo: Avaliar a prevalência de transtornos mentais não psicóticos (TMNP) e fatores associados em pessoas hipertensas e/ou diabéticas oriundas de Unidades de Saúde da Família de Blumenau, Santa Catarina. Métodos: Trata-se de um estudo transversal com 710 usuários de Unidades da Saúde da Família de Blumenau selecionados após amostragem probabilística, estratificada e por conglomerados. Os selecionados foram submetidos a um questionário domiciliar por entrevistadores treinados e responderam questões pertinentes a características socioeconômicas, pessoais e de medicamentos. 0 instrumento utilizado para o rastreamento de TMNP foi o Self-Report Questionnaire-20 (SRQ-20). As variáveis estudadas foram submetidas à análise uni e multivariada além de outros testes estatísticos adequados a cada uma delas. Resultados: A prevalência de TMNP encontrada foi de 39,44\%. Fatores associados com TMNP, após analise multivariada, foram: sexo feminino ( $R P=1,50 ; 1,12-2,01$ IC95\%; $p<0,01)$, uso de psicotrópicos ( $R P=1,74 ; 1,34$ 2,26 IC95\%; $p<0,00)$, hospitalização e/ou comparecimento ao pronto-socorro no último ano (RP=1,30; 1,02-1,66 IC95\%; $p<0,03$ ) e classe de consumo E da classificação da Associação Brasileira de Empresas de Pesquisa (ABEP) (RP=2,62; 1,09-6,27IC95\%; $p<0,03)$. Conclusões: A grande prevalência de TMNP, relacionados principalmente às mulheres e às pessoas em condições sociais desfavoráveis, suscita uma intervenção organizada e efetiva, enquanto o tratamento adequado da doença de base e a detecção precoce dos TMNP implicariam melhor uso dos recursos de saúde pública.

\footnotetext{
Abstract

Objective: Evaluate the prevalence of non-psychotic mental disorders (NPMD) and associated factors in people with hypertension and/ or diabetes from family health units in Blumenau, Santa Catarina, Brazil. Methods: Cross-sectional study, conducted in Blumenau, with 710 selected users, after probability sampling, and stratified by conglomerates, from family health basic units. Those selected were submitted to a questionnaire at home by trained interviewers and answered questions about socioeconomic, personal characteristics

Médico; Doutor em Ciências pela Faculdade de Medicina da Universidade de São Paulo (USP); Professor do Departamento de Medicina da Universidade Regional de Blumenau (FURB), Blumenau (SC), Brasil.

Acadêmicos do Curso de Medicina da FURB, Blumenau (SC), Brasil.

Endereço para correspondência: Ramiro Vieira, Universidade Regional de Blumenau, Centro de Ciências da Saúde, Rua Antonio da Veiga, 140, CEP: 89012-900 - Victor Konder, Blumenau (SC), Brasil, Telefone: (47) 3321-0243, E-mail: vieirache@gmail.com
} 
and medicines. The instrument used to screen NPMD was the Self-Report Questionnaire-20 (SRQ-20). The variables were submitted to univariate and multivariate analysis and other statistical tests appropriate to each variable studied. Results: The prevalence of NPMD was $39.44 \%$. Factors associated with NPMD after multivariate analysis were: female (RP=1.50, 1.12-2.01Cl 95\%, $\mathrm{p}<0.01$ ), use of psychotropic drugs ( $\mathrm{RP}=1.74,1.34-2.26 \mathrm{Cl} 95 \%, \mathrm{p}<0.00$ ), hospitalization and/or show the emergency medical services in the last year ( $\mathrm{RP}=1.30$, 1.02-1.66Cl 95\%, p<0.03) and E class of consumption in the Associação Brasileira de Empresas de Pesquisa (ABEP, in English Brazilian Association of Market Research Companies) classification (RP=2.62, 1.09-6.27Cl 95\%; $\mathrm{p}<0.03$ ). Conclusions: The high incidence of NPMD, mostly related to women and to people with lower socioeconomic conditions, raises an organized and effective intervention, while the appropriate treatment of base disease and early detection of NPMD mean better use of public health resources.

\section{Introdução}

Os transtornos mentais não-psicóticos (TMNP) são compostos pelos episódios de humor, transtornos de humor, transtornos por abuso de substâncias e transtornos de ansiedade, alimentares e somatoformes ${ }^{1}$.

Estudos epidemiológicos mostram que milhões de pessoas sofrem algum tipo de doença mental no mundo e que este número vem aumentando progressivamente, principalmente nos países em desenvolvimento ${ }^{2}$. Entretanto, apenas uma pequena parte deles é identificada e tratada ${ }^{3}$. Estudos apontam a dificuldade dos médicos, em especial dos generalistas, de diagnosticá-los e tratá-los ${ }^{4,5}$.

Estudos populacionais urbanos estimaram a prevalência de TMNP em maiores de 15 anos entre 17 e 36\%3,6-8. Estudos realizados com usuários de Postos da Saúde da Família (PSF) urbanos, com idade superior a 14 anos, encontraram variação de 24,9 a $35 \%^{8,9,10}$.

Fatores pessoais e socioeconômicos geralmente relacionados à TMNP são: sexo feminino ${ }^{6,7,8,10}$, idade acima $50 \operatorname{anos}^{6-9}$, estado civil divorciado/separado/viúvo ${ }^{6,7}$, presença de doença crônica ${ }^{6}$, consumo de álcool ou tabaco ${ }^{6}$, pouco apoio social ${ }^{7}$, duas ou menos consultas no último ano ${ }^{6}$. Já os fatores socioeconômicos envolvidos são: baixa escolaridade ${ }^{3,6,8,10}$, baixa renda familiar ${ }^{3,6-8,10}$, classe $\mathrm{D}$ e $\mathrm{E}^{6}$, desemprego ${ }^{8}$, emprego instá$\mathrm{vel}^{8}$. Os TMNP não estão estatisticamente relacionados com: hipertensão arterial sistêmica (HAS), diabetes mellitus (DM), índice de massa corporal (IMC) e local de consulta, público ou privado ${ }^{6,10}$. Somam-se a isso fatores estressantes crônicos como: ser vítima de abuso físico ou sexual crônico, doença grave em filho ou em si mesmo, dificuldades significativas com o empregador, criar filho sozinho (pai ou mãe), residir em zona de alta criminalidade e insatisfação no trabalho, que também se associam com transtornos mentais ${ }^{11}$.

Nos Estados Unidos, os transtornos de ansiedade são os mais comuns e debilitantes de todas as doenças mentais, muito associados à diminuição no rendimento laboral ${ }^{12}$. Também há forte evidência da associação entre pressão san- guínea e ansiedade ${ }^{13}$. A depressão está associada a danos funcionais, diminuição da produtividade, aumento da utilização do sistema de saúde e aumento no risco de suicídio. Nos Estados Unidos, os custos diretos e indiretos da depressão são estimados em 43,7 bilhões de dólares ${ }^{5}$.

Pessoas com ansiedade têm mais chance de ter HAS quando comparadas às sem ansiedade, no mínimo 3,6 vezes. No entanto, depressão grave está associada a baixo risco de hipertensão arterial no homem e esse achado não está presente em mulheres ${ }^{13}$.

Ainda há problemas psicossociais e ambientais que podem afetar o diagnóstico, o tratamento e o prognóstico dos transtornos mentais como: problemas relacionados ao grupo primário de apoio, ao ambiente social, problemas educacionais, ocupacionais, de moradia, econômicos, com acesso aos serviços de saúde, relacionados com o sistema judicial e outros ${ }^{14}$.

O objetivo deste trabalho foi estudar a prevalência de TMNP e fatores associados em pessoas com DM e/ou HAS atendidas em unidades de PSF em Blumenau, Santa Catarina.

\section{Metodologia}

Este artigo é parte integrante do estudo "Adesão ao tratamento farmacológico de pacientes com hipertensão arterial e/ou diabetes mellitus em Unidades de Saúde da Família em Blumenau, Santa Catarina”.

Trata-se de um estudo transversal tendo como unidade amostral pessoas com HAS e/ou DM.

Foram incluídas na pesquisa pessoas com HAS e/ou DM cadastradas na área de abrangência das unidades de saúde da família do município de Blumenau, Santa Catarina, com pelo menos seis meses de acompanhamento clínico, em unidades de saúde da família implantadas há, no mínimo, um ano antes do início do trabalho de campo, que teve início em abril de 2006. Algumas pessoas cadastradas como hipertensas não eram acompanhadas pela equipe de saúde da família, mas por médico de convênio de saúde da empresa em que trabalhava ou outros, sendo também incluídas. 
Procedeu-se com amostragem por conglomerados em dois estágios. No primeiro estágio, foi realizada amostragem aleatória simples de 10 unidades de saúde da família (dentre as 34 existentes que estavam em atividade há mais de 12 meses). No segundo estágio amostral, foram identificadas aquelas pessoas moradoras da área de abrangência das unidades que foram cadastradas como hipertensas, partindo-se então para amostragem aleatória estratificada, distribuindo o tamanho amostral proporcionalmente às pessoas com HAS cadastradas em cada unidade.

O tamanho da amostra foi calculado considerando uma exposição ao fator de risco de $45 \%$ nos expostos e $40 \%$ nos não-expostos, uma razão de 2 na relação não exposto/exposto de modo a se estimar os resultados com intervalo de $95 \%$ de confiança e um poder de estudo de $80 \%$. Assim, obteve-se uma amostra de 546 pessoas, a qual foi multiplicada por 1,5 para corrigir o fator do desenho (amostra por conglomerados em 2 estágios). A amostra final foi proposta em 819 pessoas. Foram consideradas 95 perdas (incluindo óbitos e mudanças de residências) ou recusas e 14 pessoas apresentaram dados incompletos sendo excluídos do estudo, constituindo uma amostra final de 710 pessoas.

A variável dependente foi a presença de TMNP. Para estimá-la, utilizou-se o Self-Reporting Questionnaire $(\text { SRQ-20) })^{15}$, um instrumento de identificação para TMNP na atenção primária, o qual é formado por 20 questões com respostas afirmativas ou negativas, cujas respostas determinam um score, com o qual será possível identificar um provável caso de TMNP. Optou-se como ponto de corte oito ou mais respostas positivas para ambos os sexos segundo proposto por Gonçalves e Kapczinski ${ }^{1}$.

As variáveis de estudo foram ordenadas em três blocos:

1. Variáveis socioeconômicas:

- escolaridade: coletada em anos de estudo completos e posteriormente agrupados em: analfabetos, 1 a 4, 5 a 8, 9 a 12 anos de estudo;

- estado ocupacional: empregado, empregador, autônomo, aposentado, desempregado ou em auxíliodoença; depois, agrupados de forma que empregado, empregador e autônomo compusessem a categoria trabalhando e os demais não trabalhando;

- classe de consumo: construída com base nos critérios da Classificação Econômica do Brasil da Associação Brasileira de Empresas de Pesquisa (ABEP) ${ }^{16}$.

2. Medicamentos:

- quantidade de medicamentos consumidos: variável discreta dada pelo número de medicamentos de todas as classes, consumidos pela pessoa há mais de uma semana comprovados pela apresentação da embalagem; depois agrupada entre aqueles que consumiam até cinco, e aqueles que consumiam seis ou mais medicações;

- consumo de psicotrópicos: medicamentos classificados sob os códigos N05 (A e B) e N06 (A, B e C) da Classificação ATC (Anatomical-Therapeutical- Chemical) ${ }^{17}$.

3. Características pessoais:

- sexo;

- idade: em anos completos e ordenada em faixa etárias de 10 anos;

- estado conjugal: casado, solteiro, viúvo ou separado, posteriormente agrupado em casado ou não-casado;

- possui religião: católica, espírita, protestante/evangélica, outra, não segue qualquer religião, depois agrupado entre possui ou não religião;

- etnia/cor: conforme referida pelo entrevistado dentre as opções branca, preta/parda, amarela, outra, depois agrupada entre branco e não-branco;

- tabagismo: se era fumante;

- consumo de álcool: com base no questionário Audit modificado, foi estimado o consumo de álcool, depois foram agrupados entre consumidor ou não de bebidas alcoólicas;

- atividades físicas: frequência de atividade física semanal, depois entre os que praticavam ou não atividade física;

- doença de base: relato da presença de HAS, ou DM ou ambas como doença de base;

- outras doenças: relato de outras doenças crônicas associadas e em tratamento, além daquelas descritas como doença de base.

- hospitalização/PS no último ano: se foi hospitalizado ou esteve no pronto-socorro no ano em que a entrevista foi realizada.

Os dados foram obtidos mediante entrevistas realizadas na residência das pessoas com HAS e/ou DM por entrevistadores previamente treinados. Os entrevistados foram informados do estudo por meio da leitura do Termo de Consentimento Livre e Esclarecido. Àqueles que concordaram em participar do estudo assinando o termo, foi aplicado um questionário elaborado para o estudo, que, por sua vez, foi submetido previamente a teste-piloto.

Todos os questionários foram revisados pelos pesquisadores para controle de qualidade do preenchimento. Uma subamostra de aproximadamente $5 \%$ dos entrevistados foi revisitada pelo pesquisador e/ou tiveram seus prontuários nas unidades de saúde consultados, com objetivo de esclarecer ou complementar as informações obtidas nas entrevistas.

Calculou-se a prevalência de transtornos mentais com intervalo de $95 \%$ de confiança. 
A associação entre TMNP e fatores associados foi expressa na forma de razão de prevalência (medida de associação) com respectivo intervalo de $95 \%$ de confiança. Inicialmente procedeu-se análise univariada com teste qui-quadrado de Pearson. No caso de variável ordinal, utilizou-se o teste quiquadrado de tendência linear.

$\mathrm{Na}$ análise multivariada, utilizou-se regressão de Poisson para se estimar a razão de prevalência. $\mathrm{O}$ modelo final foi construído pelo método "stepwise backward", em que todas as variáveis que apresentaram um valor de $\mathrm{p} \leq 0,10$ na análise univariada foram incluídas no modelo, sendo retiradas passo a passo aquelas que foram perdendo significância estatística. $\mathrm{O}$ ajuste do modelo final foi testado pelo método "Goodness of fit" ${ }^{18}$.

Foi aceito um nível de significância de p<0,05.

\section{Resultados}

A prevalência de TMNP encontrada na população estudada foi de 39,44\% (IC95\% 35,8 - 43,1).

Em relação às características pessoais e socioeconômicas, verificou-se que a população do estudo consistia majoritariamente de

Tabela 1: Distribuição das variáveis socioeconômicas

\begin{tabular}{|lcc}
\hline Variáveis & $\begin{array}{c}\text { Frequência } \\
\text { absoluta (n) }\end{array}$ & $\begin{array}{c}\text { Frequência } \\
\text { relativa (\%) }\end{array}$ \\
\hline Escolaridade & & \\
\hline $\begin{array}{l}\text { Ensino médio, superior completo ou } \\
\text { incompleto }\end{array}$ & 66 & 9,31 \\
\hline Ensino médio completo ou incompleto & 114 & 16,08 \\
\hline Ensino fundamental completo ou incompleto & 432 & 60,93 \\
\hline Não-alfabetizado & 97 & 13,68 \\
\hline Estado ocupacional & & \\
\hline Trabalhando & 207 & 29,15 \\
\hline Não trabalhando & 503 & 70,85 \\
\hline Classe de consumo & & 0 \\
\hline A1 & 0 & 0,14 \\
\hline A2 & 1 & 2,82 \\
\hline B1 & 20 & 14,23 \\
\hline B2 & 101 & 50 \\
\hline C & 355 & 31,55 \\
\hline D & 224 & 1,27 \\
\hline E & 9 & \\
\hline
\end{tabular}

Tabela 2: Distribuição das variáveis de medicamentos

\begin{tabular}{lcc}
\hline Variáveis & $\begin{array}{c}\text { Frequência } \\
\text { absoluta (n) }\end{array}$ & $\begin{array}{l}\text { Frequência } \\
\text { relativa (\%) }\end{array}$ \\
\hline $\begin{array}{l}\text { Quantidade de remédios por dia } \\
\text { Até cinco }\end{array}$ & 523 & 77,6 \\
\hline $\begin{array}{l}\text { Seis ou mais } \\
\text { Consumo de psicotrópicos }\end{array}$ & 151 & 22,4 \\
\hline Não & 132 & 18,59 \\
Sim & 578 & 81,41 \\
\hline
\end{tabular}

mulheres, brancas, entre 45 e 69 anos, casadas, com ensino fundamental completo ou incompleto, aposentadas e pertencentes às classes $\mathrm{C}$ e $\mathrm{D}$ da classificação da ABEP. A maioria não declarou não ingerir álcool, não fumar, ser sedentária, hipertensa, possuir outra doença concomitantemente e consumir até cinco medicações por dia, incluindo psicotrópicos (Tabelas 1, 2 e 3).

Após análise univariada, as variáveis não-associadas estatisticamente ao TMNP foram: idade, raça/cor, estado conjugal, religião, tabagismo, consumo de álcool, atividade física, doença de base, outras doenças, escolaridade, estado ocupacional e quantidade de remédios consumidos por dia.

Tabela 3: Distribuição das variáveis pessoais

\begin{tabular}{|c|c|c|}
\hline Variáveis & $\begin{array}{l}\text { Frequência } \\
\text { absoluta (n) }\end{array}$ & $\begin{array}{l}\text { Frequência } \\
\text { relativa (\%) }\end{array}$ \\
\hline \multicolumn{3}{|l|}{ Sexo } \\
\hline Masculino & 224 & 31,55 \\
\hline Feminino & 486 & 68,45 \\
\hline \multicolumn{3}{|l|}{ Idade (anos) } \\
\hline até 39 & 50 & 7,04 \\
\hline de 40 a 49 & 124 & 17,46 \\
\hline de 50 a 59 & 223 & 31,41 \\
\hline de 60 a 69 & 159 & 22,39 \\
\hline de 70 a 79 & 124 & 17,46 \\
\hline 80 ou mais & 30 & 4,23 \\
\hline \multicolumn{3}{|l|}{ Estado conjugal } \\
\hline Casado & 463 & 65,4 \\
\hline Não-casado & 245 & 34,6 \\
\hline \multicolumn{3}{|l|}{ Religião } \\
\hline Possui & 699 & 98,73 \\
\hline Não possui & 9 & 1,27 \\
\hline \multicolumn{3}{|l|}{ Etnia/Cor } \\
\hline Branco & 576 & 81,13 \\
\hline Não-branco & 134 & 18,87 \\
\hline \multicolumn{3}{|l|}{ Tabagismo } \\
\hline Sim & 102 & 14,37 \\
\hline Não & 608 & 85,63 \\
\hline \multicolumn{3}{|l|}{ Consumo de álcool } \\
\hline Sim & 194 & 27,32 \\
\hline Não & 516 & 72,68 \\
\hline \multicolumn{3}{|l|}{ Atividade física } \\
\hline $\operatorname{Sim}$ & 211 & 29,72 \\
\hline Não & 499 & 70,28 \\
\hline \multicolumn{3}{|l|}{ Doença de base } \\
\hline Diabetes mellitus (DM) & 103 & 14,51 \\
\hline Hipertensão arterial (HAS) & 341 & 48,03 \\
\hline Ambas & 266 & 37,46 \\
\hline \multicolumn{3}{|l|}{ Outras doenças } \\
\hline Sim & 426 & 60,08 \\
\hline Não & 283 & 39,92 \\
\hline \multicolumn{3}{|c|}{ Hospitalização/pronto-socorro no último ano } \\
\hline $\operatorname{Sim}$ & 221 & 31,17 \\
\hline Não & 488 & 68,83 \\
\hline \multicolumn{3}{|c|}{ Transtorno mental não-psicótico (TMNP) } \\
\hline $\operatorname{Sim}$ & 280 & 39,44 \\
\hline Não & 430 & 60,56 \\
\hline
\end{tabular}


Dentre as variáveis submetidas à análise univariada, aquelas que demonstraram significância estatística seguem abaixo na Tabela 4.

Após análise multivariada, as variáveis sexo, uso de psicotrópicos, hospitalização no último ano e classe econômica E permaneceram com significância estatística (Tabela 5).

\section{Discussão}

A prevalência de TMNP em pessoas com HAS e/ou DM encontrada em Blumenau é alta, superior às pesquisas realizadas por Costa et al. em Pelotas ${ }^{6}$, Maragno et al. em São Paulo $^{10}$, Ludermir e Melo Filho em Olinda ${ }^{3}$, e Gianini et al. em Sorocaba9 ${ }^{9}$. Uma das hipóteses para essa alta prevalência é justamente a composição exclusiva da amostra por portadores de doenças crônicas.

Destaca-se que este é, provavelmente, o primeiro estudo com população de atenção primária em que toda a amostra é portadora de doenças crônicas, 37\% tem mais que duas doenças, características já associadas com TMNP no trabalho de Costa et al. ${ }^{6}$

Outra hipótese aventada é que a prevalência elevada pode estar ligada a questões culturais e étnicas da amostra, que, como já foi demonstrado, pode interferir nos levantamentos epidemiológicos psiquiátricos ${ }^{19,20}$.

A alta prevalência não pode ser justificada pelo instrumento de rastreamento utilizado (SRQ-20) visto que foi empregado em vários locais com resultado menor. A primeira validação do SRQ-20 no Brasil foi realizada na década de 1980 e teve sensibilidade de $83 \%$ e especificidade de $80 \%$, utilizando como ponto de corte $7 / 8$ para mulheres e $5 / 6$ para homens $^{15}$. Após 20 anos, o SRQ-20 foi comparado com o Structured Clinical Interview for DSM-IV-TR, e verificou-se que ele é capaz de rastrear os episódios de humor, transtornos de humor, transtornos de ansiedade e somatoformes; porém, é incapaz de rastrear transtornos alimentares e os transtornos por abuso de substâncias. Além disso, estipulou-se novo ponto de corte de $7 / 8$, independentemente do sexo. Nesse escore, a sensibilidade para presença de transtorno mental não-psicótico foi de $86,33 \%$ e a especificidade, de $89,31 \%^{1}$.

Um dos motivos apontados para a diferença do ponto de corte menor para os homens naquela época deve-se ao comportamento dos homens em relação a problemas psicoemocionais e não a problemas inerentes ao instrumento. $\mathrm{Ou}$ seja, homens teriam mais dificuldades em expressar questões emocionais e pessoais de qualquer natureza, pois seriam socialmente entendidas como fraqueza ${ }^{21}$.

Além disso, o primeiro ponto de corte utilizado no SRQ-20 foi de $7 / 8$ para mulheres e $5 / 6$ para homens, o que resultou numa prevalência alta, de 43,2\%. Porém, após aná-
Tabela 4: Variáveis associadas ao TMNP* na análise univariada

\begin{tabular}{|c|c|c|c|c|c|c|}
\hline \multirow{2}{*}{ Categoria } & \multirow{2}{*}{ Variável } & \multicolumn{2}{|c|}{ TMNP } & \multirow{2}{*}{$\mathrm{RP}^{\star *}$} & \multirow{2}{*}{ IC 95\% } & \multirow{2}{*}{ Valor de $\mathrm{p}$} \\
\hline & & Sim & Não & & & \\
\hline \multirow{2}{*}{ Sexo } & Masculino & 60 & 164 & 1 & & \\
\hline & Feminino & 220 & 266 & 1,69 & & 0 \\
\hline \multirow{2}{*}{$\begin{array}{l}\text { Quantidade de } \\
\text { remédios por dia }\end{array}$} & Até cinco & 191 & 332 & 1 & & \\
\hline & Seis ou mais & 77 & 74 & 1,4 & $1,07-1,82$ & 0,01 \\
\hline \multirow{2}{*}{$\begin{array}{l}\text { Consumo de } \\
\text { psicotrópicos }\end{array}$} & Não & 195 & 383 & 1 & & \\
\hline & Sim & 85 & 47 & 1,91 & & 0 \\
\hline \multirow{2}{*}{$\begin{array}{l}\text { Hospitalização/ } \\
\text { pronto-socorro } \\
\text { no último ano }\end{array}$} & Não & 173 & 315 & 1 & & \\
\hline & Sim & 107 & 114 & 1,37 & & 0,01 \\
\hline \multirow{3}{*}{$\begin{array}{l}\text { Estado } \\
\text { ocupacional }\end{array}$} & Sim & 63 & 144 & 1 & & \\
\hline & Não & 217 & 286 & 1,42 & & 0,02 \\
\hline & Classes A e B & 33 & 89 & 1 & & \\
\hline \multirow{3}{*}{$\begin{array}{l}\text { Classe de } \\
\text { consumo }\end{array}$} & Classe C & 140 & 215 & 1,46 & & \\
\hline & Classe D & 101 & 123 & 1,67 & & \\
\hline & Classe E & 6 & 3 & 2,46 & & 0,00013 \\
\hline
\end{tabular}

* TMNP: Transtorno mental não-psicótico; **RP: Razão de prevalências; ${ }^{* \star}$ Teste qui-quadrado de tendência linear.

Tabela 5: Variáveis associadas ao TMNP após analise multivariada

\begin{tabular}{|c|c|c|c|c|c|c|}
\hline \multirow{2}{*}{ Categoria } & \multirow{2}{*}{ Variável } & \multicolumn{2}{|c|}{ TMNP } & \multirow{2}{*}{ RR } & \multirow{2}{*}{ IC 95\% } & \multirow{2}{*}{ Valor de $\mathrm{p}$} \\
\hline & & Sim & Não & & & \\
\hline \multirow{2}{*}{ Sexo } & Masculino & 60 & 164 & 1 & & \\
\hline & Feminino & 220 & 266 & 1,5 & $1,12-2,01$ & 0,01 \\
\hline \multirow{2}{*}{$\begin{array}{l}\text { Consumo de } \\
\text { psicotrópicos }\end{array}$} & Não & 195 & 383 & 1 & & \\
\hline & Sim & 85 & 47 & 1,74 & $1,34-2,26$ & 0 \\
\hline \multirow{2}{*}{$\begin{array}{l}\text { Hospitalização/ } \\
\text { pronto-socorro no } \\
\text { último ano }\end{array}$} & Não & 173 & 315 & 1 & & \\
\hline & Sim & 107 & 114 & 1,3 & $1,02-1,66$ & 0,03 \\
\hline \multirow{4}{*}{ Classe de consumo } & Classes A e B & 33 & 89 & 1 & & \\
\hline & Classe C & 140 & 215 & 1,37 & $0,93-2,00$ & 0,11 \\
\hline & Classe D & 101 & 123 & 1,47 & $0,99-2,18$ & 0,06 \\
\hline & Classe E & 6 & 3 & 2,62 & $1,09-6,27$ & 0,03 \\
\hline
\end{tabular}

Razão de Verossimilhança: 42,6 ; RR: risco relativo; p<0,0001; TMNP: Transtorno mental não-psicótico.

lise de estudo comparativo de Gonçalves et al..$^{20}$, utilizou-se novo ponto de corte de $7 / 8$, independentemente do sexo, o qual resultou em prevalência de 39,44\%. Apesar dessa mudança no ponto de corte reduzir aproximadamente 4 pontos percentuais, a prevalência ainda permaneceu superior àquelas encontradas pelos estudos supracitados.

Este estudo encontrou associação entre TMNP e sexo feminino, conforme grande parte da literatura, apesar de diferenças regionais ${ }^{6-8,10,13}$.

Essa associação pode ser atribuída à dinâmica das relações de poder entre os sexos que pode resultar em opressão para as mulheres ${ }^{22}$, o que está relacionado, segundo vários autores, ao desequilíbrio entre dominação e subordinação econômica e social ${ }^{3,23}$.

Com relação à associação do TMNP com variáveis socioeconômicas, o fato de o indivíduo não estar trabalhando, assim como pertencer a classes de consumo C, D e E mostraram-se associadas na análise univariada. Muitos estudos relacionam a baixa escolaridade aos TMNP ainda que nes- 
te estudo não se tenha encontrado significância estatística. Porém, após análise multivariada, somente a variável classe de consumo se manteve, o que pode indicar uma associação entre essas variáveis.

Ainda cumpre lembrar que a escolaridade aumenta a possibilidade de escolhas na vida, além de influenciar aspirações, autoestima e aquisição de novos conhecimentos, que podem motivar atitudes e comportamentos mais saudáveis. Dessa maneira, influencia as condições socioeconômicas futuras e sua inserção na estrutura ocupacional, logo, seu papel social. Levando-se em conta tais considerações, a sua falta diminui o poder de decisão do indivíduo, gerando uma relativa incapacidade de influenciar o meio e, consequentemente, gerar dano à saúde psicológica ${ }^{3,22}$.

A associação entre hospitalização ou comparecimento ao pronto-socorro no último ano e TMNP, tomado como indicador de consumo de serviço em caso de complicação de doenças crônicas, não foi analisada em nenhum outro artigo.

A dificuldade de detecção e tratamento dos TMNP pelo generalista, associado à deficiência no acompanhamento especializado dos TMNP e das doenças crônicas, pode resultar em tratamento inadequado com possíveis descompensações dos pacientes ${ }^{4,5}$

As pessoas com TMNP têm uma predisposição a consumir mais medicamentos, principalmente psicotrópicos, devido à presença de sintomas, principalmente nos casos graves de ansiedade, independentemente do sexo ${ }^{13}$.

Conforme os resultados, não há associação entre TMNP e HAS ou DM, o que se mostra consistente com a literatura examinada $^{6,10}$.

Além disso, pacientes hipertensos, com ou sem diabetes, podem ter redução dos sintomas ansiosos quando usam betabloqueadores podendo mascarar os dados. Porém, pacientes hipertensos que não fazem tratamento ou não o fazem de maneira adequada têm mais sintomas de ansiedade ${ }^{13}$.

Este estudo pode apresentar limitações. Ao avaliar os fatores não-associados, a amostra de estudo foi planejada para avaliar adesão ao tratamento farmacológico, sendo que o número de indivíduos pode ter sido insuficiente para resultar numa associação estatística significante com outras variáveis.

\section{Conclusão}

Nossos achados alertaram para a ocorrência de uma grande prevalência de TMNP em pessoas com HAS e/ou DM atendidas nas unidades de saúde da família, em especial em mulheres em condições sociais desfavoráveis, consumidoras de serviços e de medicamentos psicotrópicos.

\section{Referências}

1. Gonçalves DM, Kapczinski F. Prevalência de transtornos mentais em indivíduos de uma unidade de referência para Programa Saúde da Família em Santa Cruz do Sul, Rio Grande do Sul, Brasil. Cad Saúde Pública. 2008;24(9):2043-53.

2. Menezes PR. Princípios de epidemiologia psiquiátrica. In: Almeida OP, Dratcu L, Laranjeira R (Org.). Manual de psiquiatria. Rio de Janeiro: Guanabara Koogan; 1996. p. 43-55.

3. Ludermir AB, Melo Filho DA. Condições de vida e estrutura ocupacional associadas a transtornos mentais comuns. Rev Saúde Pública. 2002;36(2):213-21.

4. Brito MES. Transtornos mentais comuns em pacientes com AIDS que fazem uso de anti-retrovirais no Estado de São Paulo, Brasil. [Dissertação]. São Paulo: Universidade de São Paulo; 2002

5. Valenstein M, Vijan S, Zeber JE, Boehm K, Buttar A. The cost-utility of screening for depression in primary care. Ann Intern Med. 2001;134(5):34560.

6. Costa JSD, Menezes AMB, Olinto MTA, Gigante DP, Macedo S, Britto MAP, et al. Prevalência de distúrbios psiquiátricos menores na cidade de Pelotas, RS. Rev Bras Epidemiol. 2002;5(2):164-73.

7. Costa AG, Ludermir AB. Transtornos mentais comuns e apoio social: estudo em comunidade rural da Zona da Mata de Pernambuco, Brasil. Cad Saúde Pública. 2005;21(1):73-9.

8. Marín-León L, Oliveira HB, Barros MBA, Dalgalarrondo P, Botega J. Social inequality and common mental disorders. Rev Bras Psiquiatr. 2007;29(3):250-3.

9. Gianini RJ, Carvalho TC, Anjos RMP, Pinto PLS, Maluf ME, Lanza LB et al. Prática de rastreamento no cenário do Programa Saúde da Família em Sorocaba (SP). Rev bras educ med. 2008;32(1):15-22.

10. Maragno L, Goldbaum M, Gianini RJ, Novaes HMD, César CLG. Prevalência de transtornos mentais comuns em populações atendidas pelo Programa Saúde da Família (QUALIS) no Município de São Paulo, Brasil. Cad Saúde Pública. 2006;22(8):1639-48.

11. Duncan BB, Schmidt MI, Giugliani ERJ (Ed). Medicina ambulatorial: condutas de atenção primária baseadas em evidências. $3^{a}$ ed. Porto Alegre: Artmed; 2004.

12. Grigsby $A B$, Anderson RJ, Freedland KE, Clouse RE, Lustman PJ. Prevalence of anxiety in adults with diabetes: a systematic review. J Psychosom Res. 2002;53(6):1053-60.

13. Paterniti S, Alpérovitch A, Ducimetiére P, Dealberto MJ, Lépine JP, Bisserbe JC. Anxiety But Not Depression Is Associated With Elevated Blood Pressure in a Community Group of French Elderly. Psychosom Med. 1999;61:77-83.

14. DSM-IV-TR: manual diagnóstico e estatístico de transtornos mentais. Trad. Claudia Dornelles. Porto Alegre: Artes Médicas; 2002.

15. Mari JJ, Williams P. A validity study of a psychiatric screening questionnaire (SRQ-20) in primary care in the city of Sao Paulo. Br J Psychiatry. 1986;148:23-6.

16. ABEP (Associação Brasileira de Empresas de Pesquisa). Critério de Classificação Econômica Brasil. [citado 2007 ago 15]. Disponível em: http://www.marketanalysis.com.br/arquivos-download/biblioteca/cceb-1. pdf

17. World Health Organization. Anatomical Therapeutic Chemical (ATC) Classification. [citado 2006 abr 4]. Disponivel em: http://www.whocc.no/ atcddd/

18. Altman DG. Practical statistics for medical research. London: Chapman \& Hall; 1991.

19. [No authors listed]. Cross-national comparisons of the prevalences and correlates of mental disorders. WHO International Consortium in Psychiatric Epidemiology. Bull World Health Organ. 2000;78(4):413-26.

20. Gonçalves DM, Stein AT, Kapczinski F. Avaliação de desempenho do SelfReporting Questionnaire como instrumento de rastreamento psiquiátrico: um estudo comparativo com o Structured Clinical Interview for DSM-IV-TR. Cad Saúde Pública. 2008;24(2):380-90.

21. Mari JJ, Williams P. Misclassification by psychiatric screening questionnaires. J Chronic Dis. 1986;39(5):371-8.

22. Coutinho ESF, Almeida Filho N, Mari JJ. Fatores de risco para morbidade psiquiátrica menor: resultados de um estudo transversal em três áreas urbanas no Brasil. Rev Psiq Clín. 1999;26(5)

23. Valla W. Educação popular, saúde comunitária e apoio social numa conjuntura de globalização. Cad Saúde Pública. 1999;15(Supl 2):7-14. 